\title{
Defining a Data Impact Tool for Design Courses
}

\begin{abstract}
Wearable product/service solutions are increasingly spreading in everyday life aiming at supporting and tracking activities, also increasing self-awareness about behaviors and body parameters. The amount and variety of personal data generated and collected by these digital services is unprecedented in human history, and impacts on individuals, societies and organizations can now be only partly predicted. The design of data management in these solutions has so become a critical task for both designers and service providers. This paper presents the results of the application of a design tool, as part of an Impact Anticipation Method, created to foresee problems and opportunities so to improve design choices in quick design processes and design courses. The tool aims at stimulating the discussion on impacts related to the use of personal information. The results point out the usefulness of the method's tool in raising awareness in designers, elicit critical thinking fostering the discussion on the topic, and provide insights for improvements. It demonstrates the need of more agile ways to address the topic in the design process. Students faced their lack of knowledge about how and when personal information is implied in their solutions, and on potential impacts that such information could have.
\end{abstract}

\section{Keywords}

Personal Information, Design Tool, Impact Anticipation Method, Ethical And Societal Impacts

\section{Introduction}

Wearable product/service solutions are increasingly spreading in everyday life aiming at supporting and tracking activities mainly for wellbeing and healthy purposes, also increasing self-awareness about behaviors and body parameters. Data-driven solutions commonly employ personal data to provide value within services, and the management of such data has become a critical task for designers and service providers. The design of a new product/service is often carried on with the purpose of responding to needs of people and communities; in other words, design for innovation is often directed toward utopian goals. However, innovative solutions and functionalities, when implemented in the society, can lead to unexpected consequences related to some elements that compose the solution, or to the use of the solution itself. As pointed out by visionary technologists and scholars [1] [2] [3] [4], the use of personal data in services has a social relevance as an agency that could have impacts on the way people perceive themselves [5][6] [7], on how the perceive the contexts they interact 
with, and on how they participate in social life [8][9] [10]. It also changes actions and behaviors [5][6][11], as well as roles and relationships between people and between people and organizations [12][13][14][15]. Ackoff's DIKW model [16] defined data as "symbols that represent the properties of objects and events and their environment". Data "are useless until they are in a useable (i.e. relevant) form" [16]. Taking persons as carriers of properties, we consider personal data as raw material to be processed so to be useful. Personal information is then the signifier extracted from the elaboration of personal data. While the authors of this paper are engaged in an extensive research about the use of personal data in design [17][18], in this paper we focus on personal data involved in wearable devices. Wearable technologies and sensors embedded in wearables gather data about individuals and process them so to create personal information that is valuable for the users and for service providers. Personal information is interpreted by services through analysis and comparison with rules, reference knowledge, statistics and algorithms; in this way, it becomes knowledge that can be used to provide added value through service functions or provide awareness to the user itself. The amount and variety of personal data that are generated and collected by digital services is unprecedented in human history, and the impacts on individuals, societies and organizations that can now be only partly predicted. From the design point of view, the management of data is therefore a new dimension of social responsibility in design, requiring suitable attention and efforts. In other words, as designers, we argue the importance of developing design approaches apt to exploit the potentials of data to produce value for individuals and communities, and, on the other hand, to learn how to predict the possible - utopian and dystopian - impacts of functionalities and services employing data, so to adopt suitable design choices.

The main research developed an approach (knowledge, method and tools) for the anticipatory investigation of the potential impacts of the management of personal data in product/service design [19][20]. In this paper we focus on the use of a light (reduced and adapted) approach, to be adopted in education and whenever the design resources do not allow the use of our main methodology. The paper reports the results obtained applying the light approach in an education workshop, and the main features of the proposed approach. The results demonstrate that the anticipation activities provide design hints and guidelines for the responsible development of services and systems.

\section{Exploring Impacts Related to Personal Data in the Design of Wearable Products/ Services}

To foresee the impacts that an interactive service could have when implemented in the society, we defined the Impact Anticipation Method (IAM). The method brings the society's point of view into the design process to encourage and support ethical discussion on future impacts of solutions that imply the collection and use of personal data and personal information. It identifies insights to raise awareness and stimulate critical thinking during the design phases enabling the identification of the potential impacts. Using this approach, for each specific project brief, we can identify critical themes related to the use of personal information that we consider as relevant for the assessment of features of a new project [19][20].

For the specific topic of this paper, we report here the critical themes that can significantly be related to the design of wearable devices involving the use of personal data. Each theme should be considered during the design process so to orient the creation of a new solution toward the optimal satisfaction of user needs and to the maximum respect of basic individual right.

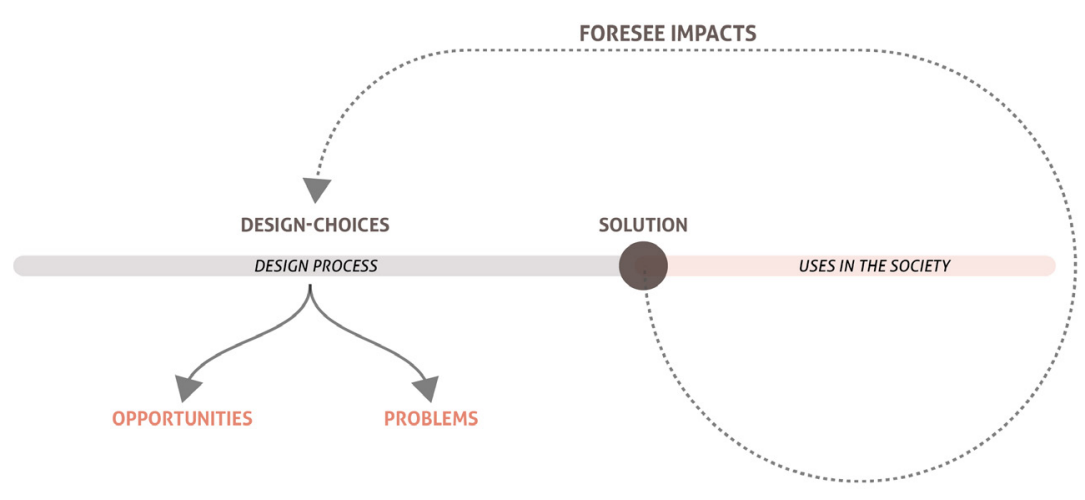

Fig. 1. The IAM aims to foresee issues (opportunities and problems) during the design process 


\section{Awareness of Data Tracking, Sharing and Use.}

The users of a wearable connected object are supposed to be well aware of detection and tracking of behaviors and biological data in real-time. What if they are not fully knowledgeable? Some issues arise when users are not completely conscious about when the tracking occurs, which data is collected, with whom every kind of data is shared or sold, who is using it and what kind of profit can be obtained by providers and partners.

Altered Cognitive Load. Proactive and reactive services can lower cognitive load allowing the user to shift the focus from tasks to the user experience. However, especially in the case of wearables, the use of the technology itself can increase the cognitive load due to information and knowledge returned as visual feedback or insights. Furthermore, wearables can raise stress due to information and suggestions related to problems, options and situations the user wasn't aware of.

Self-mirroring into Data. The users' self-perception changes and relates to the self-knowledge they acquire while understanding their own's data [21]. When the users access the data, their ability to conveniently interpret the returned information is crucial with respect to the improvement of self-knowledge and to avoid misinterpretation. Services should therefore provide the information consistently with the user's interpretation skills.

Altered Attitude and Quality of Life. Tailoring and automation can improve already existing services and, as well, enable the creation of new ones for specific purposes. The increasing availability and pervasive use of sensors can change users' attitude in actions and behaviors. People behave in a different way when they know (or think) they're being observed [22]. A system can however take advantages from the 'observer effect' aiming at changing users' behavior for their good according to goal settings.

\section{Creation and Management of Community of} Value. The interaction of people through their data often creates or reinforce a community thanks to the sharing of values related to the purpose of data tracking. As an instance, a community of runners can sort out clusters based on running performance. Crucial tasks are to ensure that the exposition of data among the community is volunteer and to consider, in the design process, possible issues related to self-exposure and self-disclosure; as well, the lack of representation in the community of people that don't track themselves should be considered.

\subsection{The Impact Anticipation Method}

The Impact Anticipation Method is the main outcome of a $\mathrm{PhD}$ research [23] and it eventually aims to elicit critical thinking in designers so to support decisionmaking during the design process of innovative solution. The raising of awareness of possible impacts, allows designers to create more robust and reliable products strengthening also the image of the company that is providing the service. The method is a twofold system: the first one consists in the Potential Issues Database aimed at the creation of knowledge about the possible consequences, - problems and opportunities - that the use of personal information in connected services might produce. The second part of IAM consists in the application of this knowledge in the design process through the Data Impact Tools so to raise awareness in the designers about possible impacts. The Potential Issues Databases is a rich collection of references, reporting situations associated to the use of personal data in services and connected products, coming from real world discussions and from future scenarios extracted from fiction. The references are classified and therefore explorable through an interactive platform [24]. The knowledge included in the Potential Issues Database ranges between different application fields and can be employed in different design contexts. Following the IAM, the impacts related to the use of personal data and information can be expressed in four layers: i) user's self-perception; ii) people's behavior and performance of actions; iii) interpersonal relationships; iv) social agency [19][20]. The iteration of the tools' design has been performed applying and refining them in use cases of research projects and design courses. This paper focuses on the second phase of the method and reports results of a research investigating the potential of the approach suitable in design contexts with string constraints of time and resources. Possible applications of the knowledge contained in the Potential Issues Database refer to four main activities of the design process: 
Analytic. In the preliminary analytic phase, designers explore the context for which they have to design the interactive solution. In this phase, the Potential Issues Database provides suitable refences through research filters (Impact Layers) of use cases and situations, so to foster the discussion on user's rights and produce design guidelines for the subsequent design phases.

Creative. Designers ideate the concept through the envisioning of context scenarios of application. In this design phase, the Potential Issues Database supports the envisioning of scenarios through the analysis of insights coming from eight macro-scenarios that we created to represent in a synthetic way the most important situations that can be associated to data management [18].

Refinement. Designers deal with specific choices (physical design) and formalize the solution through prototyping and refinements. The knowledge contained in the database support the decision-making about functionalities. The application of the Data Impact Tool in this phase is the specific focus of this paper.

Assessment. The assessment phase allows designers to evaluate the solution and test it with users through prototypes. The Potential Issues Database supports the evaluation of features and the preparation and conduction of user tests so to center the attention on ethical and social elements to create guidelines for future iterations of the project.

\section{A Light Approach to the Investigation of Impacts Related to the Use of Personal Information}

Design tools are the means by which designers preside over the cornerstones of the process, so to ensure that all the most critical and important aspects are nevertheless carried forward and considered. Designers use visualization to develop design thinking, to share knowledge with project partners, to create consensus with stakeholders, and to guide the development and implementation of the material parts and those not tangible of a system [25][26]. In other words, design tools are a designer way to produce knowledge and to support the suitable development of projects. The IAM approach is not just focused on the production of

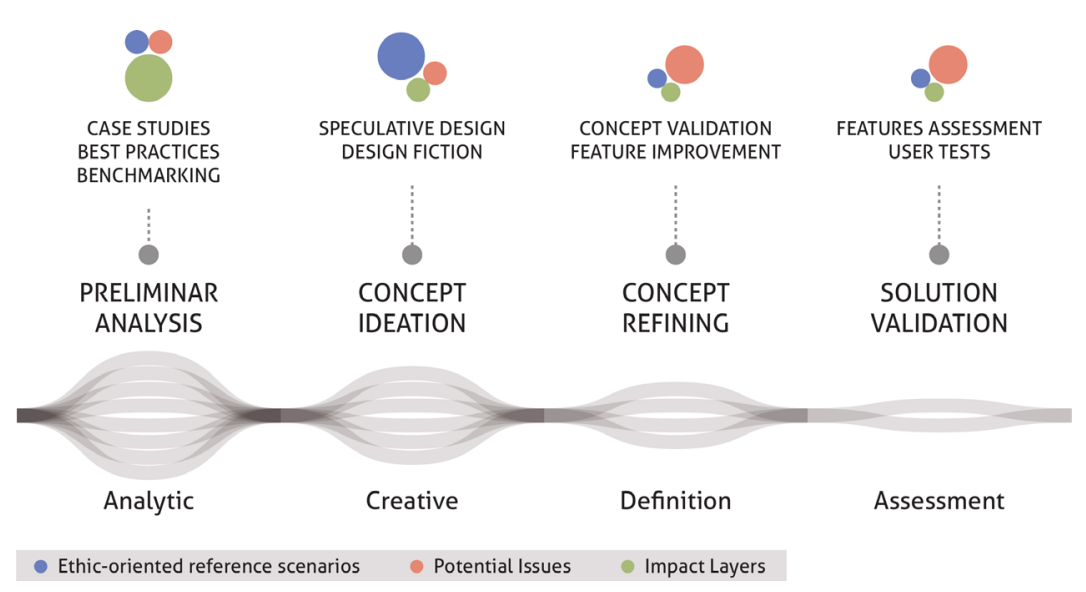

Fig. 2. Application of the knowledge of the Potential Issues

Database in the design process

knowledge, but our research also includes experiments to investigate how the knowledge can be applied through design tools in different contexts. For these reasons, we have developed a light tool to be used in design education and in the design-contexts with limited resources.

\subsection{The Creation of the Light Data Impact Tool}

In previous work we described some applications of the method [19] and its iteration [20] that led to the creation of an In-depth Data Impact Tool meant to be used by expert designers in complex and long design processes. However, the validation of the In-depth Data Impact Tool highlighted limits related to its complexity and time needed for its use. We created the Light Data Impact Tool that is meant for the design contexts where the full data-base is not available or when the time and resources for design are limited, consists in some design activities, specifically focused on the investigating

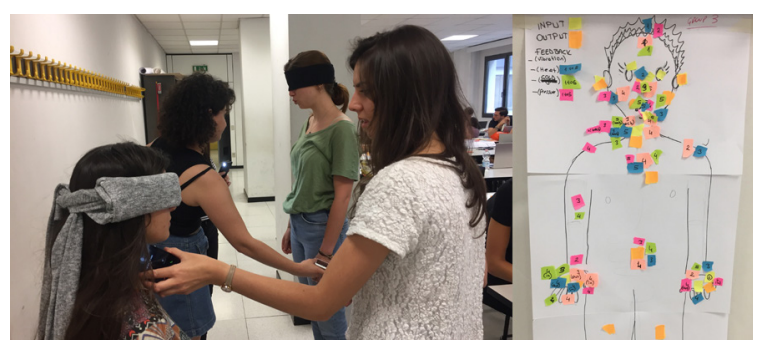

Fig. 3. Students working on on-body sensations and related body map of stimuli used as reference for the creation of the concept 
the potentials and criticalities connected to the use of personal data for a specific design brief. The features of the light tool are explained in the following of the paper through the report of its application in an education experiment.

\subsection{The Workshop About On-Body Interaction Design}

The workshop "In Close Proximity" was conducted by Professor Patrizia Marti and Laura Varisco at Politecnico di Milano in June 2018 with a class of students (around 40 ) of the master's degree program in Digital and Interaction Design as a reflection on the experience of use of on-body technologies [27]. It guided the students through a research-through-design exploration following a step-by-step process to design wearable solutions, from the initial idea to the interaction dynamics using various design tools (e.g. Body Map, Mood board, Personas, Bodystorming, Wizard of Oz, interviews, and questionnaires).

The students dedicated one full day of the two-week workshop to the exploration of possible impacts of the use of personal information of their designed solutions. We used this workshop to validate an agile Light Data Impact Tool that can be use in education and by professional designers. The tool has been used during the definition phase, when designers already had a clear concept for their solution and were defining features and details. The application of the tool followed four steps:

Step 1 - Lecture. A lecture to clarify the topic of the use of personal information, and the related types of opportunities and problems.

Step 2 - Identification of Implied Personal Information. A template was distributed, and students had 30 minutes to fill it with: A written

\begin{tabular}{|l|l|}
\hline Session 1 (Teams 2,4,6,8) & Session 2 (Teams 1,3,5,7) \\
\hline (1h) Group conversation & (1h) Template 2 fulfillment \\
\hline (1h) Template 2 fulfillment & (1h) Group conversation \\
\hline
\end{tabular}

Table 1. Organization of the activities for the two sessions description of how and why their solution implies personal information; The identification of all the actors involved in the service and their motivation; The list of technologies implied to gather and manage personal information.

Step 3 - Exploration of the Potential Issues. In order to have comparable results, we divided the 8 teams of students in two sessions. The first session spent one-hour in a conversation leaded by one of the teachers, informing them about the Potential Issues Database. After the conversation, students had one hour to fill a second template, reporting elements related to the consequences of the use of personal information that they identified as relevant for their concept. The second session of students performed the same activities, but at first, they fulfilled the second template without the support of the Potential Issues Database, and, later, they had the group conversation (Table 1). We compared the outputs (templates produced with and without the support of the knowledge contained in the Potential Issues Database) so to verify the usefulness of the conversation on issues.

\section{Step 4 - Selection of Issues and Discussion of} Improvements. As a last step, we asked the students to select, for their own project, the issues they considered as most relevant and worth of discussion. We also asked them to list the improvements they would propose for their concept in a third template. At the end of the day, we collected student feedbacks in a questionnaire about the activities performed. According to the answers, the students found useful both the content and the discussion.

\section{Results of the Use of the Light Data Impact Tool}

The results of the workshop, together with some further experiments, confirmed the usefulness of the IAM to orienting designers toward improvements of their concepts, and to raise awareness about the consequences of their design choices. Furthermore, the workshop produced evidence of the effectiveness of the light tool, since: i) all the students stated that the lecture and the group discussion allowed them to find interesting correlations between the use of personal data and possible impacts; ii) the interactive exploration 
of the Light Potential Issues Database [28], organizes in critical themes [20], reduces the complexity related to the amount of information allowing designers to nimbly access the knowledge saving time and effort; iii) the students were able to improve their projects considering aspects related to the privacy and rights of the user (pointing out the advantages of avoiding storage of unnecessary data, and the importance of giving users the control on data sharing to avoid the perception of being exposed - e.g. deciding to allow users to clog a camera while not used - Fig. 4), but also including aspects related to impacts that goes beyond privacy concerns such as avoiding overload of information through careful redesign feedbacks from the system (e.g. reducing the amount notifications, deleting data after 4 hours, and creating interaction summaries in the form of abstract artworks that aggregates data avoiding useless details and comparison between involved users - Fig. 5); iv) although many of the improvements focused on avoiding problems, others aimed at increasing features of the concept as result of discussing about opportunities given by the use of personal data. Working on eating experience for people with impaired taste [29], a team proposed that the service could consider user's food preferences to provide additional features. Another team, working on an automatic translator for slang and common language phrases [30], considered storing translated sentences to allow users to recall them when needed.

\section{Conclusion}

The validation activities and feedback from students provided useful insights about strengths and criticalities, supporting the Light Data Impact Tool and the IAM method approach producing evidences about two topics: First, the results of the identification of personal information involvement in the concepts, show that students struggled in understanding what personal data their concepts use, how the information flows work in their solution and what are the involved actors. They have little prior awareness about the role played by personal information in their solution, and about the criticalities and potentials. The introduction of a tool for representing data and their flows in the system, enables thinking and addresses the attention on this subject. Secondly, the students had difficulty on exploring the numerous issues and clusters of information contained

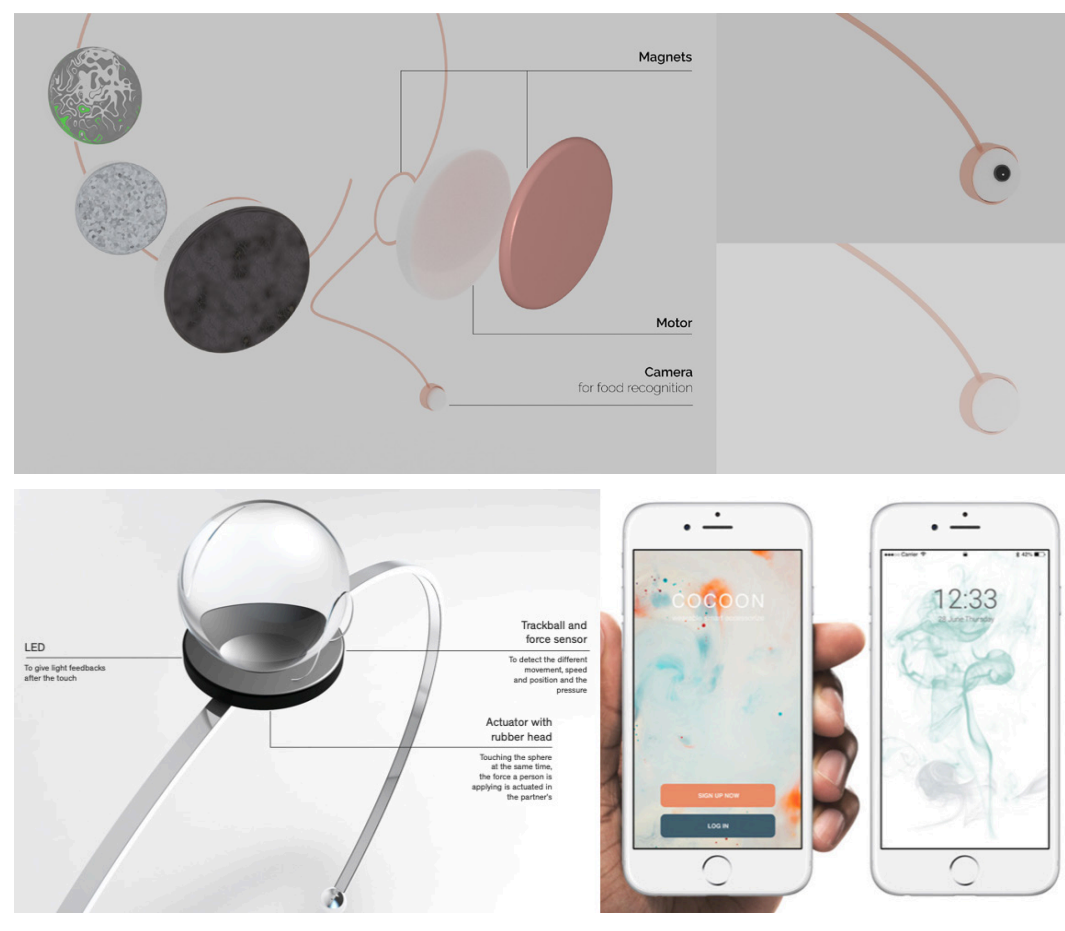

Fig. 4. (above)Tasteless' [29] is a project of a neckless that imply image recognition for food experience. After the use of the tool, the students included the possibility to clog the camera while not used so to protect the user's privacy concept.

Fig. 5. (below) 'Cocoon' [31] is a project of a bracelet aiming to improve physical communication between people in long distance relationships. The use of the tool led the students to carefully design notification mechanisms so to preserve meaning while avoiding information overload.

in the Potential Issues Database; the knowledge generated by the research is huge and difficult to access. On one hand the observation and the students' responses to a further online survey related to the activities and during informal interviews, point out the importance and usefulness of the development of the Light Potential Issues Database as a valid tool to support their awareness on both the use of personal information and its possible consequences. On the other hand, they demonstrate the need of agile ways to address the topic in the design process. Students faced their lack of knowledge about how and when personal information is implied in their designed solutions, and on potential impacts that the use of such information and the derived knowledge could have. This made clear that there is a need of critical thinking for the design of solutions implying the management of personal data. The design of tools that help designers in envisioning and foresee 
impacts demonstrates to be a valid contribution for the design culture to allow designers in creating responsible solutions that consider ethical and social issues in the design process and stimulate the conversation about responsibility in the design field.

\section{Acknowledgements. This work has been partially} funded by TIM S.p.A., Service Innovation Department, Joint Open Lab S-Cube, Milano, Italy.

\section{References}

1. Weiser. M. (1999). The computer for the 21st century. ACM SIGMOBILE Mob. Comput. Commun. Rev., vol. 3, no. 3. (pp. 3-11).

2. Bell, G. \& Dourish, P. (2007). Yesterday's tomorrows: notes on ubiquitous computing's dominant vision. Pers. Ubiquitous Comput., vol. 11, no. 2. (pp. 133-143).

3. Shapiro, A.N. (2004). Star Trek: technologies of disappearance. Berlin: Avinus.

4. Lanier, J. (2013). Who owns the future?, First Simon \& Schuster hardcover edition. New York: Simon \& Schuster.

5. Neff G. \& Nafus D. (2016). Self-tracking. Cambridge, Massachusetts: The MIT Press.

6. Young, N. (2013). The virtual self: how our digital lives are altering the world around us. Plattsburgh, NY: McClelland \& Stewart.

7. Li, I., Dey, A.K., \& Forlizzi, J. (2011) Understanding my data, myself: supporting self-reflection with ubicomp technologies. In Proceedings of the 13th international conference on Ubiquitous computing - UbiComp '11, Beijing, China. (p. 405).

8. Martani, C., Lee D., Robinson, P., Britter, R., \& Ratti, C. (2012) ENERNET: Studying the dynamic relationship between building occupancy and energy consumption. Energy Build., vol. 47. (pp. 584-591).

9. Mitchell, W.J. (2010). Me++: the cyborg self and the networked city. Cambridge, Mass: MIT.

10. Goodchild, M.F. (2007). Citizens as sensors: The world of Volunteered Geography. GeoJournal, vol. 69, no. 4. (pp. 211-221).

11. Joinson, A., Reips, U. D., Buchanan, T. \& Schofield, C.B.P. (2010) Privacy, Trust, and Self-Disclosure Online. Hum.Comput. Interact., vol. 25, no. 1. (pp. 1-24)

12. Frost J. H. \& Massagli, M.P. (2008). Social Uses of Personal Health Information Within PatientsLikeMe, an Online Patient Community: What Can Happen When Patients Have Access to One Another's Data. J. Med. Internet Res., vol. 10, no. 3. (p. e15)

13. Bisker S. \& Casalegno, F. (2009) Crowd Computing for Some: Weaving Threads of Privacy through Public Spaces. presented at the Crowd Computer Interaction Workshop in CHI 2009. (p. 4)

14. Metaxas, G., Markopoulos, P. \& Aarts, E.H.L. (2012). Modelling social translucency in mediated environments. Univers. Access Inf. Soc., vol. 11, no. 3. (pp. 311-321).

15. Lupi G. \& Posavec. S. (2016). Dear data. London UK: Particular Books.

16. Ackoff, R.L. (1989) From data to wisdom. J. Appl. Syst. Anal., vol. 16, no. 1.

17. Pillan, M., Varisco, L., \& Bertolo, M. (2017). Facing Digital Dystopias: A Discussion about Responsibility in the Design of Smart Products. In Proceedings of the Conference on Design and Semantics of Form and Movement - Sense and Sensitivity, DeSForM 2017, Alonso M. B. \& Ozcan, E. Eds. InTech.

18. Pillan, M. \& Bertolo, M. (2017). Personal digital trails: toward a convenient design of products and services employing digital data. In 4D Design Development Developing Design Conference Proceedings, Kaunas University of Technology. (p. 10).

19. Varisco, L., Pavlovic, M. \& Pillan, M. (2019) Anticipating Ethical Elements When Designing Al Agents That Employ Personal Data. In HCl International 2019 [in publishing].

20. Varisco, L., Colombo, S. \& Casalegno, F. (2019). Designing with Data. Anticipating the Impact of Personal Data Usage on Individuals and Society. In Intelligent Human Systems Integration 2019, vol. 903, Karwowski W. \& Ahram, T. Eds. Cham: Springer International Publishing. (pp. 870-876).

21. Arda, Z. (2014). Designing Identities on the Digital Mirrors of Facebook: The Reflection \& the Real. In Proceedings of the 5th STS Italia Conference. (pp. 495-509).

22. McCarney, R., Warner, J., lliffe, S., van Haselen, R., Griffin, M. \& Fisher, P. (2007). The Hawthorne Effect: a randomised, controlled trial. BMC Med. Res. Methodol., vol. 7, no. 1.

23. Varisco, L. (2019) Personal Information Design: Introducing the discussion on the consequences of the use of personal information in the design process, PhD Dissertation, Politecnico di Milano, Milan, Italy.

24. Varisco, L. (2018) In-dept Potential Issues Database. Accessed May 6, 2019 at https://public.tableau.com/profile/ laura.varisco\#!/vizhome/ERS_issues/lssuesDatabase

25. Kalbach, J. (2016). Mapping experiences: a guide to creating value through journeys, blueprints and diagrams. O'Reilly Media, Inc. 
26. Buxton, B. (2011). Sketching user experiences: getting the design right and the right design. Nachdr. Amsterdam: Morgan Kaufmann.

27. Wilde D. \& Marti, P. (2018). Exploring Aesthetic Enhancement of Wearable Technologies for Deaf Women. in Proceedings of the 2018 on Designing Interactive Systems Conference 2018 - DIS '18, Hong Kong, China. (pp. 201-213).

28. Varisco, L. (2018) Light Potential Issues Database. Accessed May 6, 2019 at https://public.tableau.com/profile/laura. varisco\#!/vizhome/CriticalThemes/Overview

29. Serin, Y., Tonts, S., Romanelli, M., Mazzali, A., Tedesco, S., \& Abbatantuono, F. (2018). Tasteless.

30. Barzaghi, C., Nazari, M., Sambinelli, C., Spanò, G., \& Xiaofeng, L. (2018). Lincoin.

31. Choi, S., Hizli, C., Interlandi, G., Panciroli, E. \& Petrolini, G. (2018). Cocoon. 